\title{
Magnetotransport properties of InSb-MnSb nanostructured films
}

\author{
Aleksey Kochura ${ }^{1, *}$, Sergey Marenkin ${ }^{2}$, Oleg Novodvorsky ${ }^{3}$, Vladimir Mikhalevsky ${ }^{3}$, Alexander Davidov ${ }^{4}$, Mikhail $^{2}$ \\ Shakhov ${ }^{5,6}$, Erkki Lahderanta ${ }^{6}$, Vasilii Zakhvalinskii ${ }^{7}$, and Alexander Kuzmenko ${ }^{1}$ \\ ${ }^{1}$ SouthWest State University, 9450 Let Oktjabrja str., Kursk, 305040, Russia \\ ${ }^{2}$ Kurnakov Institute of the general and inorganic chemistry of the RAS, 31 Leninskii prosp., 119991, Moscow, Russia \\ ${ }^{3}$ Institute on Laser and Information Technologies, RAS, 1 Svyatoozerskaya ul., Shatura, Moscow region, 140700 Russia \\ ${ }^{4}$ P.N. Lebedev Physical Institute of the RAS, 53 Leninskiy prosp., 119991, Moscow, Russia \\ ${ }^{5}$ A. F. Ioffe Physico-Technical Institute, 26 Politekhnicheskaya, St Petersburg 194021, Russian Federation \\ ${ }^{6}$ Department of Mathematics and Physics, Lappeenranta University of Technology, PO Box 20, FIN-53852 Lappeenranta, Finland \\ ${ }^{7}$ Belgorod National Research University, 85 Pobedy St, Belgorod, 308015, Russia
}

\begin{abstract}
Hybrid nanostructured InSb - MnSb films were obtained by the pulsed laser deposition using the mechanical droplet separation. Films structure was characterized by different methods (electron diffraction, scanning electron microscopy, atomic and magnetic force microscopy). The negative magnetoresistance (nMR) takes place below $100 \mathrm{~K}$. This temperature is several times more than the temperature at which the nMR occurs in homogenous $\operatorname{In}_{1-\mathrm{x}} \mathrm{Mn}_{\mathrm{x}} \mathrm{Sb}$ films. At low temperatures the spin-dependent scattering of the holes by the localized $\mathrm{Mn}^{2+}$ moments prevails. When the temperature rises, the low $\mathrm{nMR}$ is observed due to the weak spin-dependent scattering on magnetic inclusions.
\end{abstract}

\section{Introduction}

Incorporating of local magnetic moments into a semiconductor matrix is one of suitable ways for a realization of manipulating by electron and spin subsystems [1]. The highest Curie temperature $\left(T_{C}\right)$ in a dilute magnetic semiconductor is about $200 \mathrm{~K}$, as found for $\mathrm{Ga}_{1-\mathrm{x}} \mathrm{Mn}_{\mathrm{x}} \mathrm{As}$ [2]. Ferromagnetic nanoclusters embedding in a nonmagnetic or paramagnetic semiconductor can help to rich the room temperature ferromagnetism. Such hybrid systems with coexisting of a ferromagnetic phase and a semiconducting matrix demonstrate interesting properties and can be used to fabricate spintronic devices [3, 4]. Incorporating of ferromagnetic nanoscale precipitations into III-V semiconductors with different dimensions: nanowires (1D), layers (2D) and bulk samples (3D) can substantially alter the observed magnetic characteristics and galvano-magnetic effects [3 - 7]. MnAs and MnSb are high temperatures ferromagnetic semimetals and formed nanoclusters with suitable structure compatibilities to III-V compounds [8]. It is shown that $\mathrm{MnAs} / \mathrm{III}-\mathrm{V}$ structures with the huge positive magnetoresistance and the robust tunnel megnetoresistance effect may find an application in high sensitivity magnetic sensors and other magneto-electric devices $[3,9]$. The critical temperature of MnAs $T_{C}=$ $317 \mathrm{~K}$ is slightly higher than the room temperature. So, $\mathrm{MnSb}$ with $T_{C}=585 \mathrm{~K}$ is a more available candidate for applications with operating temperatures of traditional electronic materials. GaSb/MnSb nanolayers were grown earlier by the molecular beam epitaxy [10 - 12], the pulsed laser deposition (PLD) [13] and the magnetron co-sputtering [14] methods. They demonstrate ferromagnetism and spin- depended scattering above the room temperature. An influence of the ferromagnetic $\mathrm{MnSb}$ inclusions on magnetic and electrical properties of $\mathrm{InSb} / \mathrm{MnSb}$ nanostructures was observed both in bulk [15-17] and in films [18, 19].

Here we report the results about structural, magnetic, electrical and magnetotransport properties of magnetic eutectic InSb-MnSb nanostructured films grown by PLD method.

\section{Results and discussion}

\subsection{Experimental results}

Thin hybrid InSb - MnSb films were obtained by PLD. The mechanical droplet separation was used to avoid the droplet deposition. In details the growth procedure is described in Ref. 13.

The InSb-MnSb system has an eutectic near 6.5 mol\% MnSb [20]. At first we grew by the Bridgman method the eutectic composition consisting of InSb single crystal matrix and faceted single-crystal $\mathrm{MnSb}$ inclusions and used it as a starting material to prepare the target.

Polished and oriented [0001] Leuco sapphire L$\mathrm{Al}_{2} \mathrm{O}_{3}$ with dimensions $4 \times 5 \mathrm{~mm}^{2}$ have been used as substrates.

Structural properties and compositions of films were examined by the Tecnai G2 20F S-T (FEI)

Corresponding author: akochura@gmail.com 
scanning transmission electron microscope (STEM) with the high angle annular dark field (HAADF) detector and the scanning electron microscope (SEM) JSM-6610LV (Jeol) with the attachment for the energy dispersive Xray spectroscopy (EDXS) X-MaxN (Oxford Instruments). The morphology and magnetic properties of films surface were determined using an AIST NT Smart SPM high resolution scanning probe microscope. Magnetization of the samples was measured in fields up to $\mathrm{B}=5 \mathrm{~T}$ at temperatures $\mathrm{T}=3-310 \mathrm{~K}$ using a superconducting quantum interference device (SQUID) magnetometer (model S600, Cryogenics ltd.). The electrical and magnetotransport properties were investigated between $4.2 \mathrm{~K}$ and $320 \mathrm{~K}$ using the standard six-point geometry in pulsed magnetic fields up to $20 \mathrm{~T}$. The resistance was measured with a high accuracy (the error was at most $1 \%$ of the measured value). The films had conductivity of p-type and linear current-voltage characteristics in the range of the measurements. The films thicknesses were about $50 \mathrm{~nm}$.

\subsection{Analysis of the results}

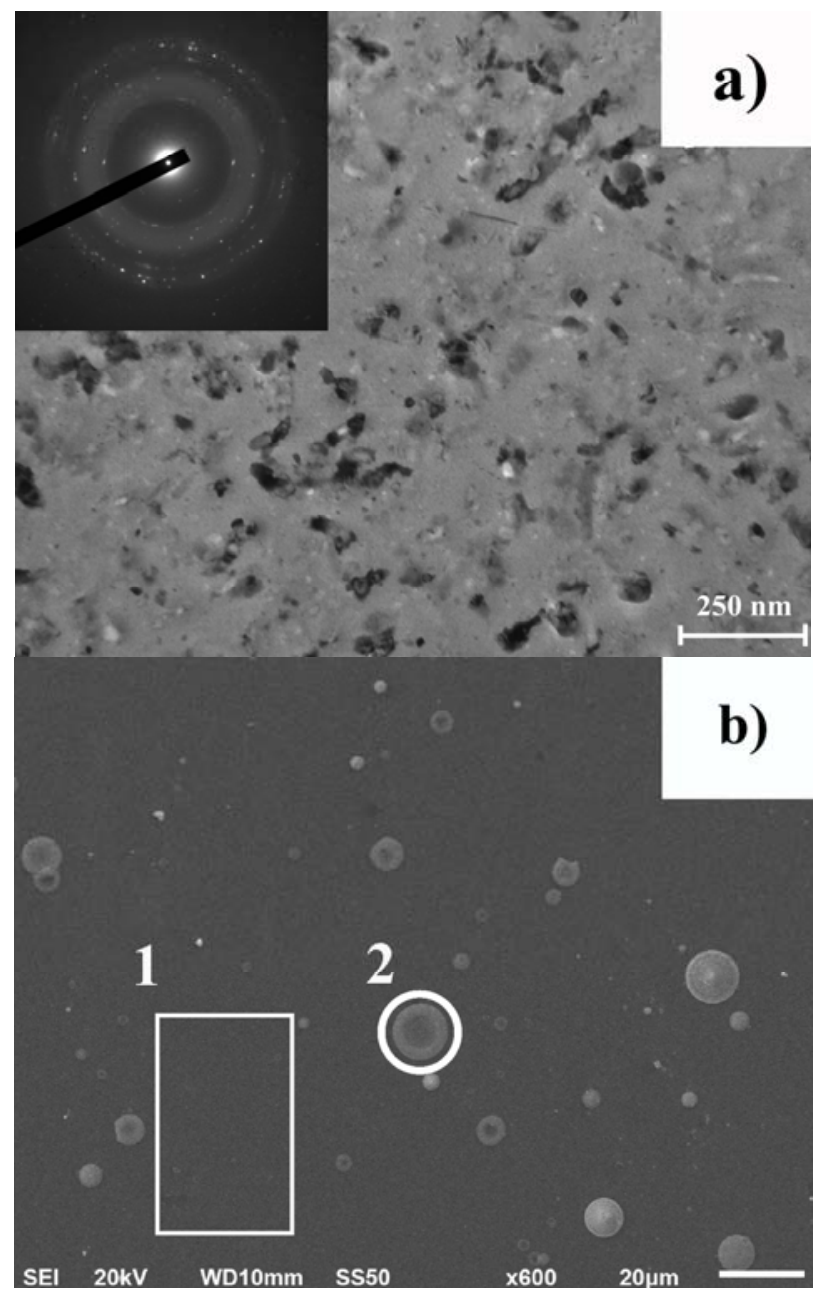

Fig. 1. Typical STEM (a) and SEM (b) images of InSb $\mathrm{MnSb}$ films. The inset a) shows a ring structure of an electron diffraction pattern. EDXS analysis was performed for two different areas (b) labeled as 1 and 2
The microstructure observation showed a non homogeneous structure of $\mathrm{InSb}$ - MnSb film in different size scales (Fig. 1). The film is polycrystalline (Fig. 1a, inset) and nanostructured (Fig. 1a) with $30-100 \mathrm{~nm}$ size of granules and nanoinclusions. Less than $10 \%$ of the surface is covered by drops with diameters from 1 to 15 mkm. All films surface contains In, Sb, Mn with the elements ratio close to a composition of the target and homogeneous distribution within EDXS size accuracy (1 $\mathrm{mkm})$. MnSb content is slightly lower only in drops (Table).

Table. A surface elemental composition of InSb$\mathrm{MnSb}$ film in different areas labelled on Fig.1b

\begin{tabular}{|c|c|c|c|}
\hline \multirow{2}{*}{ Area } & \multicolumn{3}{|c|}{ At. percent } \\
\cline { 2 - 4 } & $\mathrm{In}$ & $\mathrm{Sb}$ & $\mathrm{Mn}$ \\
\hline 1 & 44.5 & 49.8 & 5.7 \\
\hline 2 & 45.7 & 49.4 & 4.9 \\
\hline
\end{tabular}
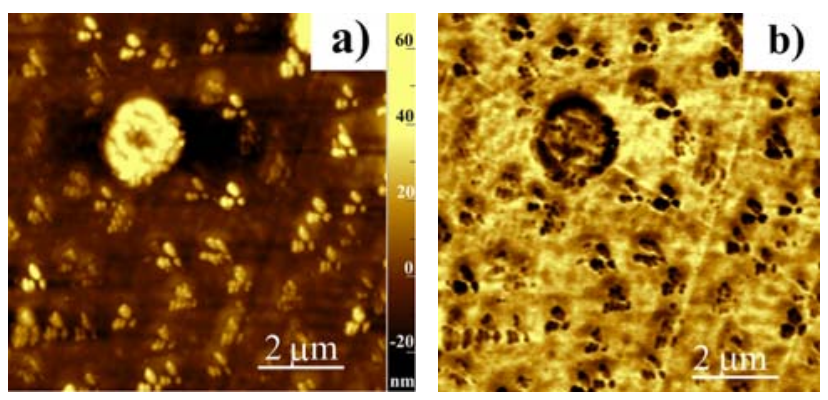

Fig. 2. Images of a site of the surface for the InSb-MnSb film obtained by the SPM in the mode of atomic force (a) and magnetic force (b) microscopy at $313 \mathrm{~K}$.

The typical images of the InSb-MnSb film surface obtained by the scanning probe microscopy (SPM) at the room temperature are given in Fig. 2. The presence of ferromagnetic inclusions is well visible. The sizes of ferromagnetic granules measured by the SPM in the magnetic force regime $(30-200 \mathrm{~nm})$ were comparable with sizes obtained from TEM (Fig. 1a).

The electrical resistivity of the film had a semiconductor character and decreased with increasing a temperature in the range from 1.6 to $300 \mathrm{~K}$. (Fig. 3). At a low temperature range the temperature dependence on resistivity is $\rho(T)=\rho_{0} \exp \left[E_{A} /(k T)\right]$, where $\rho_{0}$ - preexponential factor, $E_{A}-$ activation energy, $k-$ Boltzmann constant. [21] So the local activation energy $E_{A} \equiv d \ln \rho / d(k T)^{-1}=0.30 \mathrm{meV}$ we can found from lowtemperature linear segment of the plots $\ln \rho$ versus $(k T)^{-1}$ (Fig 3, Inset). It is in a good agreement with $E_{A}=0.28$ meV observed earlier in [22] for $p-\operatorname{InSb}(\mathrm{Mn})$ monocrystals at $N_{\mathrm{Mn}}=1.6 \cdot 10^{17} \mathrm{~cm}^{-3}$.

The field dependences of magnetoresistance $\left(\Delta \rho / \rho=\left(\rho_{B^{-}}-\rho_{0}\right) / \rho_{0}\right)$ of the InSb-MnSb film at different temperatures are shown in Fig. 4. The maximal negative magnetoresistance $\Delta \rho / \rho \approx-20 \%$ was observed at $T=4.2 \mathrm{~K}$. At $T_{n}<100 \mathrm{~K}$ magnetoresistance consisted of a negative component (nMR) prevailing up to $20 \mathrm{~T}$, increased with increasing of a magnetic field and tended to the saturation at low temperatures. Note that $T_{n}$ is 
several times more than the temperature at which the nMR is occur in homogenous $\operatorname{In}_{1-x} \mathrm{Mn}_{\mathrm{x}} \mathrm{Sb}$ films [23].

The scattering of spin-polarized carriers on isolated $\mathrm{Mn}^{2+}$ ions at temperatures as below as above $T_{C}$ leads to decreasing resistivity. When $T<T_{C}$ ferromagnetic ordered regions increase as $T$ decreases but some of fractions of the spins can still remain outside ferromagnetic network and serve as a source of magnetic scattering [23]. In this case magnetoresistance is proportional both to the spin polarization $P$ arising from the ferromagnetic block and to magnetization of the isolated paramagnetic moments $\Delta \rho / \rho \sim-4 P M(B)$ [23].

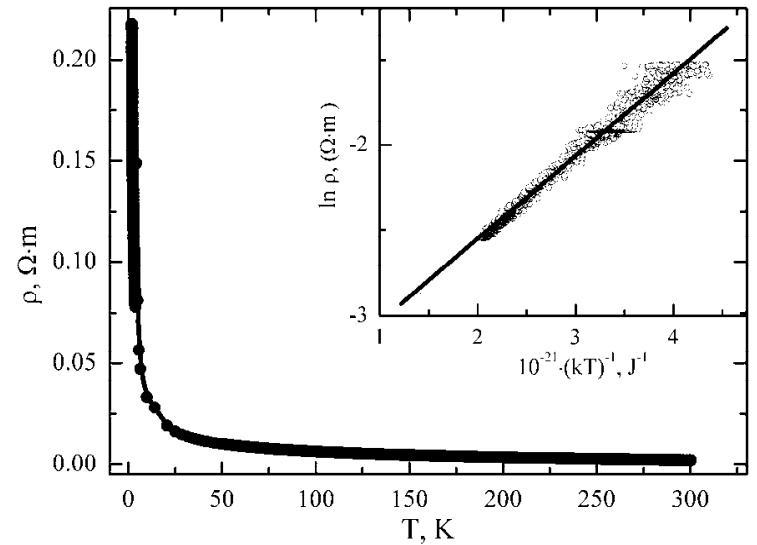

Fig. 3. A temperature dependence of the resistivity of InSb-MnSb film. Inset: linear segment of the plots of $\ln \rho$ versus $(k T)^{-1}$

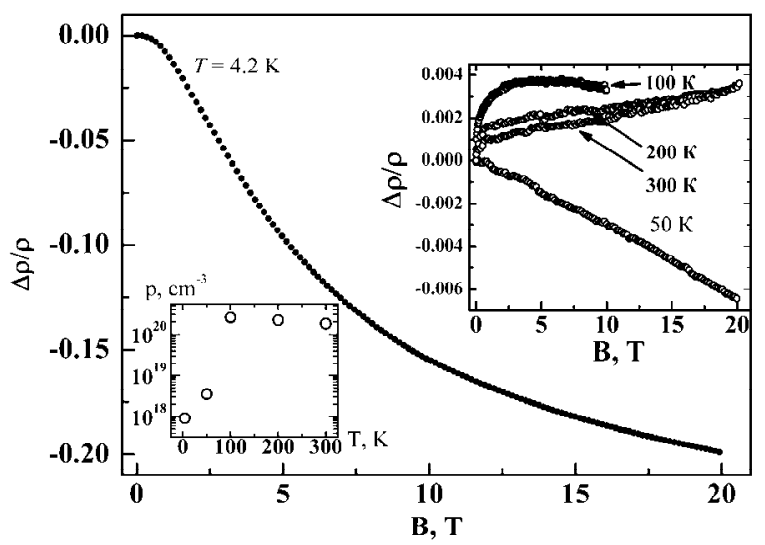

Fig. 4. A magnetic field dependence of the magnetoresistance for $\mathrm{InSb}-\mathrm{MnSb}$ film at $\mathrm{T}=4.2 \mathrm{~K}$. Left inset: The plots of the hole concentration versus temperature. Right inset: Magnetoresistance as a function of magnetic field for different temperatures.

The second approach to a description of the nMR in our compounds is similar to one for granular paramagnetic-ferromagnetic GaAs:Mn/MnAs hybrid layers with nano-scaled MnAs clusters embedded in GaAs:Mn matrix [24]. Total magnetoresistance in these systems is formed by competition of negative and positive terms and paramagnetism of matrix play the sufficient role. At low temperatures in the external magnetic field $\mathrm{B}_{\mathrm{ext}}=0$ the holes are trapped near cluster interface due to the local giant Zeeman splitting and form a bound magnetic polarons (it is accompanied by decreasing hole concentration with decreasing of a temperature (fig. 4 , the left inset). When $B_{\text {ext }}=0$ giant Zeeman splitting spreads into paramagnetic GaAs:Mn matrix resulting in aligning of carrier spins and occurring of the negative magnetoresistance. A source of the positive magnetoresistance (pMR) in granular paramagnetic-ferromagnetic systems is a difference of a spin orientation of clusters and majority carriers [25]. The thermal disorder destroys the preferential spin orientation of the $\mathrm{Mn}$ ions and the positive magnetoresistance decreases for higher temperatures.

As temperature increases the magnetoresistance follows the second scenario: negative magnetoresistance decreases, becomes positive, reaches the maximum and then slowly decreases (Fig 4, right inset). The same behavior with smaller pMR was observed for (In,Mn)Sb crystals containing microsized MnSb clusters [26] and with the same and bigger pMR for GaAs:Mn/MnAs [26, 27]. At the same time pMR depended on both a size and a distribution of clusters. For clusters lining up perpendiculary to the current pMR reached $800 \%$ and increased with decreasing clusters volumes [27, 28].

This work was supported by the Ministry of Education and Science of the Russian Federation (№ 16.2814.2017/PCh) and Russian Foundation for Basic Research (15-07-06081-a).

\section{References}

1. T. Dietl, H. Ohno, Rev. Mod. Phys., 86, 187 (2014).

2. L. Chen, X. Yang, F. Yang, J. Zhao, J. Misuraca, P. Xiong, S. von Molnar, Nano Lett., 11, 2584 (2011).

3. M.T. Elm, S. Hara, Advanced Materials, 26, 8079 (2014).

4. S. Hara, K. Komagata, Physica Status Solidi (b), 252, 1925 (2015).

5. K. Kabamoto, R. Kodaira, S. Hara, Journal of Crystal Growth, 464, 80 (2017).

6. S.F. Marenkin, A.D. Izotov, I.V. Fedorchenko, V.M. Novotortsev, Russian Journal of Inorganic Chemistry, 60, 295 (2015).

7. M. Khalid, S. Prucnal, M.O. Liedke, K. Gao, S. Facsko, W. Skorupa, M. Helm, S. Zhou, Materials Research Express, 1, 026105 (2014).

8. T. Dietl, K. Sato, T. Fukushima, A. Bonnani, M. Jamet, A. Barski, S. Kuroda, M. Tanaka, P. N. Hai, H. Katayama-Yoshida, Rev. Mod. Phys., 87, 1311 (2015).

9. P.N. Hai, Sh. Ohya, M. Tanaka, S.E. Barnes, S. Maekawa, Nature, 458, 489 (2014).

10. J. Choi, J. Choi, S. Choi, S.C. Hong, S. Cho, M.H. Sohn, Y. Park, K.-W. Lee, H.-M. Park, J.H. Song, J.B. Ketterson. Journal of the Korean Physical Society, 47, S497 (2005).

11. E. Dynowska, J. Bak-Misiuk, P. Romanowski, J.Z. Domagala, J. Sadowski, T. Wojciechowski, S. Kret, B. Kurowska, A. Kwiatkowski, W. Caliebe. Radiation Physics and Chemistry, 80, 1051 (2011). 
12. W. Braun, A. Trampert, V.M. Kaganer, B. Jenichen, D.K. Satapathy, K.H. Ploog. Journal of Crystal Growth, 301-302, 20 (2007).

13. S.F. Marenkin, O.A. Novodvorsky, A.V. Shorokhova, A.B. Dasvydov, B.A. Aronzon, A.V. Kochura, I.V. Fedorchenko, O.D. Khramova, A.V. Timofeev, Inorganic Materials, 50, 973 (2014).

14. J.A. Calderon, F. Mesa, A. Dussan, Applied Surface Science, 396, 1113 (2017).

15. K. Ganesan, S. Mariyappan, H.L. Bhat, Sol. St. Com., 143, 272 (2007).

16. A.V. Kochura, B.A. Aronzon, K.G. lisunov, A.V. Lashkul, A.A. Sidorenko, R. De Renzi, S.F. Marenkin, M. Alam, A.P. Kuzmenko, E. Lahderanta. J. Appl. Phys., 113083905 (2013).

17. E. Lahderanta, A.V. Lashkul, A.V. Kochura, K.G. Lisunov, B.A. Aronzon, M.A. Shakhov, Phys. Status Solidi A 211, 991 (2014).

18. L. Lari, S. Lea, C. Feeser, B.W. Wessels, V.K. Lasarov, J. Appl. Phys., 111, 07C311 (2012).

19. J. Liu, M.P. Hanson, J.A. Peters, B.W. Wessels, ACS Applied Materials and Interfaces, 7, 24159 (2015).

20. V.M. Novotortsev, A.V. Kochura, S.F. Marenkin, I.V. Fedorchenko, S.V. Drogunov, A. Lashkul, E. Lahderanta, Russian Journal of Inorganic Chemistry, 56, 1951 (2011).

21. B. I. Shklovskii, A. L. Efros. Electronic Properties of Doped Semiconductors (Berlin: Springer. 1984).

22. S.A. Obukhov, S.W. Tozer, W.A. Coniglio. Scientific Reports, 5, 13451 (2015).

23. M. Csontos, T. Wojtowicz, X. Liu, M. Dobrovolska, B. Janko, J.K. Furduna, G. Mihaly Phys.Rew.Lett., 95, 227203 (2005).

24. G. Zarand, C.P. Moca, B. Janko, Phys.Rev.Lett., 94, 247202 (2005).

25. A. Milner, A. Gerber, B. Groisman, M. Karpovsky, A. Gladkikh. Phys.Rev.Lett., 76, 475 (1996).

26. K. Kuzmina, B.A. Aronzon, A.V. Kochura, A.V. Lashkul, K.G. Lisunov, E. Lahderanta, M.A. Shakhov. EPJ Web of Conferencies, 75, 05014 (2014)

27. W. Heimbrodt, P.J. Klar, S. Ye, M. Lampalzer, C.Michel, S.D. Baranovskii, P. Thomas, W. Stolz, J.Supercond, 18, 315 (2005).

28. C. Michel, M.T. Elm, B. Goldlucke, S.D. Baranovskii, P. Thomas, W. Heimbrodt, P.J. Klar. Appl.Phys.Lett., 92, 223119 (2008). 